\title{
On positive solutions for a class of singular nonlinear fractional differential equations
}

\author{
Mohamed Jleli and Bessem Samet ${ }^{*}$
}

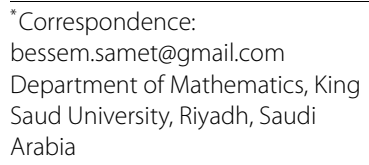

\begin{abstract}
We study the existence and uniqueness of a positive solution for the singular nonlinear fractional differential equation boundary value problem

$$
\begin{aligned}
& D_{0^{+}}^{\alpha} u(t)=f(t, u(t), u(t)), \quad 0<t<1, \\
& u(0)=u(1)=u^{\prime}(0)=u^{\prime}(1)=0,
\end{aligned}
$$

where $3<\alpha \leq 4$ is a real number, $D_{0^{+}}^{\alpha}$ is the Riemann-Liouville fractional derivative and $f:(0,1] \times[0,+\infty) \times[0,+\infty) \rightarrow[0,+\infty)$ is continuous, $\lim _{t \rightarrow 0^{+}} f(t, \cdot, \cdot)=+\infty(f$ is singular at $t=0$ ). Our approach is based on a coupled fixed point theorem on ordered metric spaces. An example is given to illustrate our main result.

MSC: $34 \mathrm{AO} 8 ; 34 \mathrm{~B} 16 ; 47 \mathrm{H} 10$
\end{abstract}

Keywords: singular fractional differential equation; positive solution; coupled fixed point; coupled lower and upper solution; ordered metric space

\section{Introduction}

Fractional differential equations arise in many engineering and scientific disciplines as the mathematical modeling of systems and processes in the fields of physics, fluid flows, electrical networks, viscoelasticity, aerodynamics, and many other branches of science. For details, see [1-11].

In the last few decades, fractional-order models are found to be more adequate than integer-order models for some real world problems. Recently, there have been some papers dealing with the existence and multiplicity of solutions (or positive solutions) of nonlinear initial fractional differential equations by the use of techniques of nonlinear analysis (fixed point theorems, Leray-Schauder theory, etc.); see [2, 4, 5, 11].

Recently, there have been many exciting developments in the field of fixed point theory on partially ordered metric spaces. The first result in this direction was given by Turinici [12]. In [13], Ran and Reurings extended the Banach contraction principle in partially ordered sets with some applications to matrix equations. The obtained result by Ran and Reurings was further extended and refined by many authors; see [14-19].

Very recently, Shurong Sun et al. [20] discussed the existence and uniqueness of a positive solution to the singular nonlinear fractional differential equation boundary value 
problem

$$
\begin{aligned}
& D_{0^{+}}^{\alpha} u(t)=f(t, u(t)), \quad 0<t<1, \\
& u(0)=u(1)=u^{\prime}(0)=u^{\prime}(1)=0,
\end{aligned}
$$

where $3<\alpha \leq 4$ is a real number, $D_{0^{+}}^{\alpha}$ is the Riemann-Liouville fractional derivative and $f:(0,1] \times[0,+\infty) \rightarrow[0,+\infty)$ is continuous, $\lim _{t \rightarrow 0^{+}} f(t, \cdot)=+\infty(f$ is singular at $t=0)$, $f(t, \cdot)$ is nondecreasing for all $t \in(0,1]$.

Motivated by the above mentioned work, in this paper we investigate the existence and uniqueness of a positive solution for the singular nonlinear fractional differential equation boundary value problem

$$
\begin{aligned}
& D_{0^{+}}^{\alpha} u(t)=f(t, u(t), u(t)), \quad 0<t<1, \\
& u(0)=u(1)=u^{\prime}(0)=u^{\prime}(1)=0,
\end{aligned}
$$

where $3<\alpha \leq 4$ is a real number, $D_{0^{+}}^{\alpha}$ is the Riemann-Liouville fractional derivative and $f:(0,1] \times[0,+\infty) \times[0,+\infty) \rightarrow[0,+\infty)$ is continuous, $\lim _{t \rightarrow 0^{+}} f(t, \cdot, \cdot)=+\infty(f$ is singular at $t=0)$, for all $t \in(0,1], f(t, \cdot, \cdot)$ is nondecreasing with respect to the first component, and it is decreasing with respect to the second component. Our approach is based on a recent coupled fixed point theorem on ordered metric spaces established by Harjani et al. [17]. We end the paper with an example that illustrates our main result.

\section{Preliminaries}

In this section, we recall some basic definitions and properties from fractional calculus theory. For more details about fractional calculus, we refer the readers to $[1,3,10]$.

Definition 2.1 The Riemann-Liouville fractional derivative of order $\alpha>0$ of a continuous function $\varphi:(0,+\infty) \rightarrow \mathbb{R}$ is given by

$$
D_{0^{+}}^{\alpha} \varphi(t)=\frac{1}{\Gamma(n-\alpha)}\left(\frac{d}{d t}\right)^{(n)} \int_{0}^{t} \frac{\varphi(s)}{(t-s)^{\alpha-n+1}} d s
$$

where $n=[\alpha]+1,[\alpha]$ denotes the integer part of number $\alpha$, provided that the right side is pointwise defined on $(0,+\infty)$.

Definition 2.2 The Riemann-Liouville fractional integral of order $\alpha>0$ of a function $\varphi$ : $(0,+\infty) \rightarrow \mathbb{R}$ is given by

$$
I_{0^{+}}^{\alpha} \varphi(t)=\frac{1}{\Gamma(\alpha)} \int_{0}^{t}(t-s)^{\alpha-1} \varphi(s) d s
$$

provided that the right side is pointwise defined on $(0,+\infty)$.

From the definition of the Riemann-Liouville derivative, we can obtain the following statement. 
Lemma 2.1 (see [10]) Let $\alpha>0$. If we assume $u \in C(0,1) \cap L(0,1)$, then the fractional differential equation

$$
D_{0^{+}}^{\alpha} u(t)=0
$$

has $u(t)=c_{1} t^{\alpha-1}+c_{2} t^{\alpha-2}+\cdots+c_{N} t^{\alpha-N}, c_{i} \in \mathbb{R}, i=1,2, \ldots, N$ as unique solutions, where $N$ is the smallest integer greater than or equal to $\alpha$.

Lemma 2.2 (see [10]) Assume that $u \in C(0,1) \cap L(0,1)$ with a fractional derivative of order $\alpha>0$ that belongs to $C(0,1) \cap L(0,1)$. Then

$$
I_{0^{+}}^{\alpha} D_{0^{+}}^{\alpha} u(t)=u(t)+c_{1} t^{\alpha-1}+c_{2} t^{\alpha-2}+\cdots+c_{N} t^{\alpha-N},
$$

for some $c_{i} \in \mathbb{R}, i=1,2, \ldots, N$, where $N$ is the smallest integer greater than or equal to $\alpha$.

The Green function of fractional differential equation boundary value problem is given by

Lemma 2.3 (see $[10])$ Let $h \in C([0,1])$ and $3<\alpha \leq 4$. The unique solution to

$$
\begin{aligned}
& D_{0^{+}}^{\alpha} u(t)=h(t), \quad 0<t<1, \\
& u(0)=u(1)=u^{\prime}(0)=u^{\prime}(1)=0,
\end{aligned}
$$

is

$$
u(t)=\int_{0}^{1} G(t, s) h(s) d s
$$

where

$$
G(t, s)= \begin{cases}\frac{(t-s)^{\alpha-1}+(1-s)^{\alpha-2} t^{\alpha-2}[(s-t)+(\alpha-2)(1-t) s]}{\Gamma(\alpha)}, & 0 \leq s \leq t \leq 1, \\ \frac{t^{\alpha-2}(1-s)^{\alpha-2}[(s-t)+(\alpha-2)(1-t) s]}{\Gamma(\alpha)}, & 0 \leq t \leq s \leq 1 .\end{cases}
$$

Here $G(t, s)$ is called the Green function of boundary value problem (3)-(4).

The following properties of the Green function will be used later.

Lemma 2.4 (see [10]) The following properties hold:

(i) $G(t, s)=G(1-s, 1-t)$ for $t, s \in(0,1)$;

(ii) $(\alpha-2) t^{\alpha-2}(1-t)^{2} s^{2}(1-s)^{\alpha-2} \leq \Gamma(\alpha) G(t, s) \leq M_{0} s^{2}(1-s)^{\alpha-2}, t, s \in(0,1)$;

(iii) $G(t, s)>0$, for $t, s \in(0,1)$;

(iv) $(\alpha-2) s^{2}(1-s)^{\alpha-2} t^{\alpha-2}(1-t)^{2} \leq \Gamma(\alpha) G(t, s) \leq M_{0} t^{\alpha-2}(1-t)^{2}, t, s \in(0,1)$, where $M_{0}=\max \left\{\alpha-1,(\alpha-2)^{2}\right\}$.

Let $(X, \preceq)$ be a partially ordered set endowed with a metric $d$ such that $(X, d)$ is complete metric space. Let $F: X \times X \rightarrow X$ be a given mapping. 
Definition 2.3 We say that $(X, \preceq)$ is directed if for every $(x, y) \in X^{2}$ there exists $z \in X$ such that $x \preceq z$ and $y \preceq z$.

Definition 2.4 We say that $(X, \preceq, d)$ is regular if the following conditions hold:

$\left(c_{1}\right)$ if $\left\{x_{n}\right\}$ is a nondecreasing sequence in $X$ such that $x_{n} \rightarrow x \in X$, then $x_{n} \preceq x$ for all $n$;

$\left(c_{2}\right)$ if $\left\{y_{n}\right\}$ is a decreasing sequence in $X$ such that $y_{n} \rightarrow y \in X$, then $y_{n} \succeq y$ for all $n$.

Example 2.1 Let $X=C([0, T]), T>0$, be the set of real continuous functions on $[0, T]$. We endow $X$ with the standard metric $d$ given by

$$
d(u, v)=\max _{0 \leq t \leq T}|u(t)-v(t)|, \quad u, v \in X
$$

We define the partial order $\preceq$ on $X$ by

$$
u, v \in X, \quad u \preceq v \quad \Longleftrightarrow u(t) \leq v(t) \text { for all } t \in[0, T] .
$$

Let $u, v \in X$. For $w=\max \{u, v\}$, that is, $w(t)=\max \{u(t), v(t)\}$ for all $t \in[0, T]$, we have $u \preceq w$ and $v \preceq w$. This implies that $(X, \preceq)$ is directed. Now, let $\left\{x_{n}\right\}$ be a nondecreasing sequence in $X$ such that $d\left(x_{n}, x\right) \rightarrow 0$ as $n \rightarrow \infty$, for some $x \in X$. Then, for all $t \in[0, T],\left\{x_{n}(t)\right\}$ is a nondecreasing sequence of real numbers converging to $x(t)$. Thus we have $x_{n}(t) \leq x(t)$ for all $n$, that is, $x_{n} \preceq x$ for all $n$. Similarly, if $\left\{y_{n}\right\}$ is a decreasing sequence in $X$ such that $d\left(y_{n}, y\right) \rightarrow 0$ as $n \rightarrow \infty$, for some $y \in X$, we get that $y_{n} \succeq y$ for all $n$. Then we proved that $(X, \preceq, d)$ is regular.

Definition 2.5 (see [15]) An element $(x, y) \in X^{2}$ is called a coupled fixed point of $F$ if $F(x, y)=x$ and $F(y, x)=y$.

Definition 2.6 (see [15]) We say that $F$ has the mixed monotone property if for all $(x, y)$, $(u, v) \in X^{2}$, we have

$$
x \preceq u, y \succeq v \quad \Longrightarrow \quad F(x, y) \preceq F(u, v) .
$$

Denote by $\Phi$ the set of functions $\varphi:[0,+\infty) \rightarrow[0,+\infty)$ satisfying:

$\left(\varphi_{1}\right) \varphi$ is continuous;

$\left(\varphi_{2}\right) \varphi$ is nondecreasing;

$\left(\varphi_{3}\right) \varphi^{-1}(\{0\})=\{0\}$.

The following two lemmas are fundamental in the proofs of our main results.

Lemma 2.5 (see [17]) Let $(X, \preceq)$ be a partially ordered set and suppose that there exists a metric $d$ on $X$ such that $(X, d)$ is a complete metric space. Let $F: X \times X \rightarrow X$ be a mapping having the mixed monotone property on $X$ such that

$$
\psi(d(F(x, y), F(u, v))) \leq \psi(\max \{d(x, u), d(y, v)\})-\varphi(\max \{d(x, u), d(y, v)\})
$$


for all $x, y, u, v \in X$ with $x \succeq u$ and $y \preceq v$, where $\psi, \varphi \in \Phi$. Suppose also that $(X, \preceq, d)$ is regular and there exist $x_{0}, y_{0} \in X$ such that

$$
x_{0} \preceq F\left(x_{0}, y_{0}\right), \quad y_{0} \succeq F\left(y_{0}, x_{0}\right) .
$$

Then $F$ has a coupled fixed point $\left(x^{*}, y^{*}\right) \in X^{2}$. Moreover, if $\left\{x_{n}\right\}$ and $\left\{y_{n}\right\}$ are the sequences in $X$ defined by

$$
x_{n+1}=F\left(x_{n}, y_{n}\right), \quad y_{n+1}=F\left(y_{n}, x_{n}\right), \quad n=0,1, \ldots,
$$

then

$$
\lim _{n \rightarrow \infty} d\left(x_{n}, x^{*}\right)=\lim _{n \rightarrow \infty} d\left(y_{n}, y^{*}\right)=0
$$

Lemma 2.6 (see [17]) Adding to the hypotheses of Lemma 2.5 the condition $(X, \preceq)$ is regular, we obtain the uniqueness of the coupled fixed point. Moreover, we have the equality $x^{*}=y^{*}$.

\section{Main result}

Let Banach space $E=C([0,1])$ be endowed with the norm $\|u\|_{\infty}=\max _{0 \leq t \leq 1}|u(t)|$. We define the partial order $\preceq$ on $E$ by

$$
u, v \in E, \quad u \preceq v \quad \Longleftrightarrow u(t) \leq v(t) \text { for all } t \in[0,1] \text {. }
$$

In Example 2.1, we proved that $(E, \preceq)$ with the classic metric given by

$$
d(u, v)=\max _{0 \leq t \leq 1}|u(t)-v(t)|, \quad u, v \in E
$$

satisfies the following properties: $(E, \preceq)$ is directed and $(E, \preceq, d)$ is regular.

Define the closed cone $P \subset E$ by

$$
P=\{u \in E: u \succeq 0\}
$$

where 0 denotes the zero function.

Definition 3.1 (see [15]) We say that $\left(u^{-}, u^{+}\right) \in C([0,1]) \times C([0,1])$ is a coupled lower and upper solution to (1)-(2) if

$$
u^{-}(t) \leq \int_{0}^{1} G(t, s) f\left(s, u^{-}(s), u^{+}(s)\right) d s, \quad \text { for all } 0 \leq t \leq 1
$$

and

$$
u^{+}(t) \geq \int_{0}^{1} G(t, s) f\left(s, u^{+}(s), u^{-}(s)\right) d s, \quad \text { for all } 0 \leq t \leq 1
$$

Our main result is the following. 
Theorem 3.1 Let $0<\sigma<1,3<\alpha \leq 4, f:(0,1) \times[0,+\infty) \times[0,+\infty) \rightarrow[0,+\infty)$ is continuous, $\lim _{t \rightarrow 0^{+}} f(t, \cdot, \cdot)=+\infty$ and $t \mapsto t^{\sigma} f(t, x, y)$ is continuous on $[0,1] \times[0,+\infty) \times[0,+\infty)$. Assume that there exists $0<\lambda \leq(1-\sigma) \Gamma(\alpha-\sigma+1) /(2 \Gamma(3-\sigma))$ such that for $x, y, z, w \in$ $[0,+\infty)$ with $x \geq z, y \leq w$ and $t \in[0,1]$,

$$
0 \leq t^{\sigma}(f(t, x, y)-f(t, z, w)) \leq \lambda \eta(\max \{(x-z),(w-y)\})
$$

where $\eta:[0,+\infty) \rightarrow[0,+\infty), \beta: u \mapsto u-\eta(u) \in \Phi$. Suppose also that (1)-(2) has a coupled lower and upper solution $\left(u^{-}, u^{+}\right) \in P \times P$. Then the boundary value problem (1)-(2) has a unique positive solution $u^{*} \in C([0,1])$. The sequences $\left\{u_{n}\right\}$ and $\left\{v_{n}\right\}$ defined by

$$
\begin{array}{ll}
u_{0}=u^{-}, & u_{n+1}=\int_{0}^{1} G(t, s) f\left(s, u_{n}(s), v_{n}(s)\right) d s, \quad n=0,1, \ldots, \\
v_{0}=u^{+}, \quad v_{n+1}=\int_{0}^{1} G(t, s) f\left(s, v_{n}(s), u_{n}(s)\right) d s, \quad n=0,1, \ldots
\end{array}
$$

converge uniformly to $u^{*}$.

Proof Suppose that $u$ is a solution of boundary value problem (1)-(2). Then

$$
u(t)=\int_{0}^{1} G(t, s) f(s, u(s), u(s)) d s, \quad t \in[0,1] .
$$

We define the operator $F: P \times P \rightarrow E$ by

$$
F(u, v)(t)=\int_{0}^{1} G(t, s) f(s, u(s), v(s)) d s, \quad t \in[0,1]
$$

- Step 1. We shall prove that

$$
F(P \times P) \subseteq P
$$

Let $u, v \in P$. Let us prove that $F(u, v) \in C([0,1])$. We have

$$
F(u, v)(t)=\int_{0}^{1} G(t, s) s^{-\sigma} s^{\sigma} f(s, u(s), v(s)) d s, \quad t \in[0,1] .
$$

By the continuity of $s^{\sigma} f(s, u(s), v(s))$ in $[0,1]$, it is easy to check that $F(u, v)(0)=0$. Now, let $t_{0} \in[0,1]$. We have to prove that

$$
\left|F(u, v)(t)-F(u, v)\left(t_{0}\right)\right| \rightarrow 0 \quad \text { as } t \rightarrow t_{0} .
$$

We distinguish three cases:

$$
t_{0}=0, \quad t_{0} \in(0,1] \text { and } t \in\left(t_{0}, 1\right], \quad t_{0} \in(0,1] \text { and } t \in\left[0, t_{0}\right) \text {. }
$$

Case 1. $t_{0}=0$. Since $s \mapsto s^{\sigma} f(s, u(s), v(s))$ is continuous on $[0,1]$, there exists a constant $M>0$ such that $\left|s^{\sigma} f(s, u(s), v(s))\right| \leq M$ for all $s \in[0,1]$. We have

$$
\left|F(u, v)(t)-F(u, v)\left(t_{0}\right)\right|=\left|\int_{0}^{1} G(t, s) s^{-\sigma} s^{\sigma} f(s, u(s), v(s)) d s\right| .
$$


Using Lemma 2.3, we have

$$
\begin{aligned}
\left|F(u, v)(t)-F(u, v)\left(t_{0}\right)\right| & \mid \int_{0}^{1} \frac{t^{\alpha-2}(1-s)^{\alpha-2}[(s-t)+(\alpha-2)(1-t) s]}{\Gamma(\alpha)} s^{-\sigma} s^{\sigma} f(s, u(s), v(s)) d s \\
& +\int_{0}^{t} \frac{(t-s)^{\alpha-1}}{\Gamma(\alpha)} s^{-\sigma} s^{\sigma} f(s, u(s), v(s)) d s \mid \\
\leq & \left|\int_{0}^{1} \frac{t^{\alpha-2}(1-s)^{\alpha-2}[(s-t)+(\alpha-2)(1-t) s]}{\Gamma(\alpha)} s^{-\sigma} s^{\sigma} f(s, u(s), v(s)) d s\right| \\
& +\left|\int_{0}^{t} \frac{(t-s)^{\alpha-1}}{\Gamma(\alpha)} s^{-\sigma} s^{\sigma} f(s, u(s), v(s)) d s\right| \\
\leq & M \int_{0}^{1} \frac{(\alpha-1) t^{\alpha-2}(1-s)^{\alpha-2}}{\Gamma(\alpha)} s^{1-\sigma} d s+M \int_{0}^{1} \frac{t^{\alpha-1}(1-s)^{\alpha-2}}{\Gamma(\alpha)} s^{-\sigma} d s \\
& +M \int_{0}^{1} \frac{(\alpha-2) t^{\alpha-1}(1-s)^{\alpha-2}}{\Gamma(\alpha)} s^{1-\sigma} d s+M \int_{0}^{t} \frac{(t-s)^{\alpha-1}}{\Gamma(\alpha)} s^{-\sigma} d s \\
= & M \frac{t^{\alpha-2}}{\Gamma(\alpha-1)} B(2-\sigma, \alpha-1)+M \frac{t^{\alpha-1}}{\Gamma(\alpha)} B(1-\sigma, \alpha-1) \\
& +M \frac{(\alpha-2) t^{\alpha-1}}{\Gamma(\alpha)} B(2-\sigma, \alpha-1)+M \frac{t^{\alpha-\sigma}}{\Gamma(\alpha)} B(1-\sigma, \alpha) \rightarrow 0 \text { as } t \rightarrow 0^{+},
\end{aligned}
$$

where $B(\cdot, \cdot)$ denotes the beta function.

Case 2. $t_{0} \in(0,1)$ and $t \in\left(t_{0}, 1\right]$. In this case,

$$
\begin{aligned}
& \left|F(u, v)(t)-F(u, v)\left(t_{0}\right)\right| \\
& =\mid \int_{0}^{1} \frac{(\alpha-1)\left(t^{\alpha-2}-t_{0}^{\alpha-2}\right)(1-s)^{\alpha-2} s^{1-\sigma}}{\Gamma(\alpha)} s^{\sigma} f(s, u(s), v(s)) d s \\
& \quad+\int_{0}^{1} \frac{\left(t_{0}^{\alpha-1}-t^{\alpha-1}\right)(1-s)^{\alpha-2} s^{-\sigma}}{\Gamma(\alpha)} s^{\sigma} f(s, u(s), v(s)) d s \\
& \quad+\int_{0}^{1} \frac{(\alpha-2)\left(t_{0}^{\alpha-1}-t^{\alpha-1}\right)(1-s)^{\alpha-2} s^{1-\sigma}}{\Gamma(\alpha)} s^{\sigma} f(s, u(s), v(s)) d s \\
& \quad+\int_{0}^{t_{0}} \frac{\left[(t-s)^{\alpha-1}-\left(t_{0}-s\right)^{\alpha-1}\right] s^{-\sigma}}{\Gamma(\alpha)} s^{\sigma} f(s, u(s), v(s)) d s \\
& \quad+\int_{t_{0}}^{t} \frac{(t-s)^{\alpha-1} s^{-\sigma}}{\Gamma(\alpha)} s^{\sigma} f(s, u(s), v(s)) d s \mid .
\end{aligned}
$$

Now, we have

$$
\begin{aligned}
& \left|F(u, v)(t)-F(u, v)\left(t_{0}\right)\right| \\
& \quad \leq M \frac{(\alpha-1)\left(t^{\alpha-2}-t_{0}^{\alpha-2}\right)}{\Gamma(\alpha)} \int_{0}^{1}(1-s)^{\alpha-2} s^{1-\sigma} d s+M \frac{\left(t^{\alpha-1}-t_{0}^{\alpha-1}\right)}{\Gamma(\alpha)} \int_{0}^{1}(1-s)^{\alpha-2} s^{-\sigma} d s \\
& \quad+M \frac{(\alpha-2)\left(t^{\alpha-1}-t_{0}^{\alpha-1}\right)}{\Gamma(\alpha)} \int_{0}^{1}(1-s)^{\alpha-2} s^{1-\sigma} d s \\
& \quad+\frac{M}{\Gamma(\alpha)} \int_{0}^{t_{0}}\left[(t-s)^{\alpha-1}-\left(t_{0}-s\right)^{\alpha-1}\right] s^{-\sigma} d s+\frac{M}{\Gamma(\alpha)} \int_{t_{0}}^{t}(t-s)^{\alpha-1} s^{-\sigma} d s
\end{aligned}
$$




$$
\begin{aligned}
= & M \frac{\left(t^{\alpha-2}-t_{0}^{\alpha-2}\right)}{\Gamma(\alpha-1)} B(2-\sigma, \alpha-1) \\
& +\frac{M\left(t^{\alpha-1}-t_{0}^{\alpha-1}\right)}{\Gamma(\alpha)}[B(1-\sigma, \alpha-1)+(\alpha-2) B(2-\sigma, \alpha-1)] \\
& +\frac{M t^{\alpha-\sigma}}{\Gamma(\alpha)} B(1-\sigma, \alpha)-\frac{M t_{0}^{\alpha-\sigma}}{\Gamma(\alpha)} B(1-\sigma, \alpha) \\
\rightarrow & 0 \quad \text { as } t \rightarrow t_{0}^{+} .
\end{aligned}
$$

Case 3. $t_{0} \in(0,1]$ and $t \in\left[0, t_{0}\right)$. The proof is similar to that of Case 2, so we omit it.

Thus we proved that $F(u, v)$ is continuous on $[0,1]$ for all $u, v \in C([0,1])$. Moreover, taking into account Lemma 2.4 and as $t^{\sigma} f(t, x, y) \geq 0$ for all $t \in[0,1], x, y \geq 0$, our claim (10) is proved. Now the mapping

$$
F: P \times P \rightarrow P
$$

is well defined.

- Step 2. We shall prove that $F$ has the mixed monotone property with respect to the partial order $\preceq$ given by (7).

Let $(x, y),(u, v) \in P \times P$ such that $x \preceq u$ and $y \succeq v$. From (8), we have

$$
s^{\sigma} f(s, x(s), y(s)) \leq s^{\sigma} f(s, u(s), v(s))
$$

for all $s \in[0,1]$. This implies that

$$
\int_{0}^{1} G(t, s) s^{-\sigma} s^{\sigma} f(s, x(s), y(s)) d s \leq \int_{0}^{1} G(t, s) s^{-\sigma} s^{\sigma} f(s, u(s), v(s)) d s,
$$

that is,

$$
\int_{0}^{1} G(t, s) f(s, x(s), y(s)) d s \leq \int_{0}^{1} G(t, s) f(s, u(s), v(s)) d s,
$$

which gives us that

$$
F(x, y)(t) \leq F(u, v)(t)
$$

for all $t \in[0,1]$, and then we have

$$
F(x, y) \preceq F(u, v) .
$$

Then $F$ has the mixed monotone property.

- Step 3. We shall prove that $F$ satisfies the contractive condition (5) for some $\psi, \varphi \in \Phi$. Let $(x, y),(u, v) \in P \times P$ such that $x \succeq u$ and $y \preceq v$. For all $t \in[0,1]$, using (8), we have

$$
\begin{aligned}
|F(x, y)(t)-F(u, v)(t)| & =\int_{0}^{1} G(t, s)[f(s, x(s), y(s))-f(s, u(s), v(s))] d s \\
& =\int_{0}^{1} G(t, s) s^{-\sigma} s^{\sigma}[f(s, x(s), y(s))-f(s, u(s), v(s))] d s
\end{aligned}
$$




$$
\begin{aligned}
& \leq \int_{0}^{1} G(t, s) s^{-\sigma} \lambda \eta(\max \{x(s)-u(s), v(s)-y(s)\}) d s \\
& \leq \lambda \eta(\max \{d(x, u), d(y, v)\}) \int_{0}^{1} G(t, s) s^{-\sigma} d s \\
& \leq \lambda \eta(\max \{d(x, u), d(y, v)\}) \max _{z \in[0,1]} \int_{0}^{1} G(z, s) s^{-\sigma} d s .
\end{aligned}
$$

Thus we have

$$
d(F(x, y), F(u, v)) \leq \lambda \eta(\max \{d(x, u), d(y, v)\}) \max _{z \in[0,1]} \int_{0}^{1} G(z, s) s^{-\sigma} d s .
$$

Now, let $z \in[0,1]$. We have

$$
\begin{array}{rl}
\int_{0}^{1} & G(z, s) s^{-\sigma} d s \\
= & \int_{0}^{1} \frac{z^{\alpha-2}(1-s)^{\alpha-2}[(s-z)+(\alpha-2)(1-z) s]}{\Gamma(\alpha)} s^{-\sigma} d s+\int_{0}^{z} \frac{(z-s)^{\alpha-1}}{\Gamma(\alpha)} s^{-\sigma} d s \\
\leq & \int_{0}^{1} \frac{(\alpha-1) z^{\alpha-2}(1-s)^{\alpha-2}}{\Gamma(\alpha)} s^{1-\sigma} d s+\int_{0}^{1} \frac{z^{\alpha-1}(1-s)^{\alpha-2}}{\Gamma(\alpha)} s^{-\sigma} d s \\
& +\int_{0}^{1} \frac{(\alpha-2) z^{\alpha-1}(1-s)^{\alpha-2}}{\Gamma(\alpha)} s^{1-\sigma} d s+\int_{0}^{z} \frac{(z-s)^{\alpha-1}}{\Gamma(\alpha)} s^{-\sigma} d s \\
\leq & \frac{z^{\alpha-2}}{\Gamma(\alpha-1)} B(2-\sigma, \alpha-1)+\frac{z^{\alpha-1}}{\Gamma(\alpha)} B(1-\sigma, \alpha-1) \\
& +\frac{(\alpha-2) z^{\alpha-1}}{\Gamma(\alpha)} B(2-\sigma, \alpha-1)+\frac{z^{\alpha-\sigma}}{\Gamma(\alpha)} B(1-\sigma, \alpha) \\
\leq & \frac{1}{\Gamma(\alpha-1)} B(2-\sigma, \alpha-1)+\frac{1}{\Gamma(\alpha)} B(1-\sigma, \alpha-1)+\frac{(\alpha-2)}{\Gamma(\alpha)} B(2-\sigma, \alpha-1) \\
& +\frac{1}{\Gamma(\alpha)} B(1-\sigma, \alpha) \\
= & \frac{2 \Gamma(3-\sigma)}{(1-\sigma) \Gamma(\alpha-\sigma+1)},
\end{array}
$$

which implies that

$$
\max _{z \in[0,1]} \int_{0}^{1} G(z, s) s^{-\sigma} d s \leq \frac{2 \Gamma(3-\sigma)}{(1-\sigma) \Gamma(\alpha-\sigma+1)}
$$

Now, using the above inequality, (11) and the fact that $\lambda<\frac{(1-\sigma) \Gamma(\alpha-\sigma+1)}{2 \Gamma(3-\sigma)}$, we get

$$
\begin{aligned}
d(F(x, y), F(u, v)) & \leq \eta(\max \{d(x, u), d(y, v)\}) \\
& =\max \{d(x, u), d(y, v)\}-\beta(\max \{d(x, u), d(y, v)\}) .
\end{aligned}
$$

Thus we proved that for all $(x, y),(u, v) \in P \times P$ such that $x \succeq u$ and $y \preceq v$, we have

$$
\psi(d(F(x, y), F(u, v))) \leq \psi(\max \{d(x, u), d(y, v)\})-\varphi(\max \{d(x, u), d(y, v)\}),
$$

where $\psi(t)=t$ and $\varphi(t)=\beta(t)$. 
- Step 4. Existence of $\left(x_{0}, y_{0}\right) \in P \times P$ such that $x_{0} \preceq F\left(x_{0}, y_{0}\right)$ and $y_{0} \succeq F\left(y_{0}, x_{0}\right)$.

We take $\left(x_{0}, y_{0}\right)=\left(u^{-}, u^{+}\right)$, the coupled lower and upper solution to (1)-(2).

Now, from Lemmas 2.5 and 2.6, there exists a unique $u^{*} \in P$ such that $u^{*}=F\left(u^{*}, u^{*}\right)$, that is $u^{*}$ is the unique positive solution to (1)-(2). The convergence of the sequences $\left\{u_{n}\right\}$ and $\left\{v_{n}\right\}$ to $u^{*}$ follows immediately from (6).

Now, we end this paper with the following example.

Example 3.1 Consider the boundary value problem

$$
\begin{aligned}
& D_{0^{+}}^{7 / 2} u(t)=\frac{(t-1 / 2)^{2}}{2 \sqrt{t}}\left(u(t)+\frac{1}{u(t)+1}\right), \quad 0<t<1, \\
& u(0)=u(1)=u^{\prime}(0)=u^{\prime}(1)=0 .
\end{aligned}
$$

In this case, $f(t, x, y)=\frac{(t-1 / 2)^{2}}{2 \sqrt{t}}\left(x+\frac{1}{y+1}\right)$, for $(t, x, y) \in(0,1] \times[0,+\infty) \times[0,+\infty)$. Note that $f$ is continuous on $(0,1] \times[0,+\infty) \times[0,+\infty)$ and $\lim _{t \rightarrow 0^{+}} f(t, \cdot, \cdot)=+\infty$. Let $\sigma=\lambda=1 / 2$ and $\eta(t)=(1 / 2) t$. For all $x, y, z, w \in[0,+\infty)$ with $x \geq z, y \leq w$ and $t \in[0,1]$, we have

$$
\begin{aligned}
0 & \leq t^{1 / 2}(f(t, x, y)-f(t, z, w)) \\
& =\frac{(t-1 / 2)^{2}}{2}[(x-z)+(w-y)] \\
& \leq(t-1 / 2)^{2} \max \{x-z, w-y\} \\
& \leq \frac{1}{4} \max \{x-z, w-y\} \\
& =\lambda \eta(\max \{x-z, w-y\}) .
\end{aligned}
$$

On the other hand,

$$
\frac{(1-\sigma) \Gamma(\alpha-\sigma+1)}{2 \Gamma(3-\sigma)}=\frac{3}{2 \Gamma(5 / 2)}=\frac{2}{\sqrt{\pi}}>\lambda=\frac{1}{2} .
$$

Consider now, the pair $\left(u^{-}, u^{+}\right) \in P \times P$ defined by $u^{-} \equiv 0$ and $u^{+} \equiv 7 / 17$. Using Lemma 2.4(iv), one can show easily that $\left(u^{-}, u^{+}\right)$is a coupled lower and upper solution to (12)-(13).

Finally, applying Theorem 3.1, we deduce that (12)-(13) has one and only one positive solution $u^{*} \in C([0,1])$.

Competing interests

The authors declare that they have no competing interests.

Authors' contributions

All authors contributed equally and significantly in writing this article. All authors read and approved the final manuscript.

Acknowledgement

This work was supported by the Research Center, College of Science, King Saud University.

Received: 29 March 2012 Accepted: 18 May 2012 Published: 12 July 2012 


\section{References}

1. Kilbas, AA, Srivastava, HM, Trujillo, JJ: Theory and Applications of Fractional Differential Equations. North-Holland Mathematics Studies, vol. 204. Elsevier, Amsterdam (2006)

2. Babakhani, A, Gejii, VD: Existence of positive solutions of nonlinear fractional differential equations. J. Math. Anal. Appl. 278, 434-442 (2003)

3. Bai, Z, Lu, H: Positive solutions for boundary value problem of nonlinear fractional differential equation. J. Math. Anal. Appl. 311, 495-505 (2005)

4. Delbosco, D, Rodino, L: Existence and uniqueness for a nonlinear fractional differential equation. J. Math. Anal. Appl. 204, 609-625 (1996)

5. Gejji, VD, Babakhani, A: Analysis of a system of fractional differential equations. J. Math. Anal. Appl. 293, 511-522 (2004)

6. Lakshmikantham, V, Vatsala, AS: Basic theory of fractional differential equations. Nonlinear Anal. 69(8), 2677-2682 (2008)

7. Nonnenmacher, TF, Metzler, R: On the Riemann-Liouville fractional calculus and some recent applications. Fractals 3 , 557-566 (1995)

8. Oldham, KB, Spanier, J: The Fractional Calculus. Academic Press, New York (1974)

9. Sabatier, J, Agrawal, OP, Machado, JAT (eds.): Advances in Fractional Calculus: Theoretical Developments and Applications in Physics and Engineering. Springer, Dordrecht (2007)

10. $X u, X$, Jiang, D, Yuan, C: Multiple positive solutions for the boundary value problem of a nonlinear fractional differential equation. Nonlinear Anal. 71, 4676-4688 (2009)

11. Zhang, S: Existence of positive solution for some class of nonlinear fractional differential equations. J. Math. Anal. Appl. 278, 136-148 (2003)

12. Turinici, M: Abstract comparison principles and multivariable Gronwall-Bellman inequalities. J. Math. Anal. Appl. 117, 100-127 (1986)

13. Ran, ACM, Reurings, MCB: A fixed point theorem in partially ordered sets and some applications to matrix equations. Proc. Am. Math. Soc. 132, 1435-1443 (2004)

14. Agarwal, RP, El-Gebeily, MA, O’Regan, D: Generalized contractions in partially ordered metric spaces. Appl. Anal. 87, 109-116 (2008)

15. Gnana Bhaskar, T, Lakshmikantham, V: Fixed point theorems in partially ordered metric spaces and applications Nonlinear Anal. 65, 1379-1393 (2006)

16. Ćirić, L, Cakić, N, Rajović, M, Ume, JS: Monotone generalized nonlinear contractions in partially ordered metric spaces. Fixed Point Theory Appl. 2008, Article ID 131294 (2008)

17. Harjani, J, López, B, Sadarangani, K: Fixed point theorems for mixed monotone operators and applications to integral equations. Nonlinear Anal. 74, 1749-1760 (2011)

18. Nieto, JJ, López, RR: Existence and uniqueness of fixed point in partially ordered sets and applications to ordinary differential equations. Acta Math. Sin. Engl. Ser. 23, 2205-2212 (2007)

19. Samet, B: Coupled fixed point theorems for a generalized Meir-Keeler contraction in partially ordered metric spaces. Nonlinear Anal. 72, 4508-4517 (2010)

20. Sun, S, Zhao, Y, Han, Z, Xu, M: Uniqueness of positive solutions for boundary value problems of singular fractional differential equations. Inverse Probl. Sci. Eng. (2011). doi:10.1080/17415977.2011.603726

doi:10.1186/1687-2770-2012-73

Cite this article as: Jleli and Samet: On positive solutions for a class of singular nonlinear fractional differential equations. Boundary Value Problems 2012 2012:73.

\section{Submit your manuscript to a SpringerOpen ${ }^{\ominus}$ journal and benefit from:}

- Convenient online submission

- Rigorous peer review

- Immediate publication on acceptance

Open access: articles freely available online

- High visibility within the field

- Retaining the copyright to your article 